\title{
p-Adic Approach to Linear 2-Normed Spaces
}

\author{
Mehmet AÇıkgöz, Nurgül Aslan, Nurten KÖşKeroĞLu, \\ AND SERKAN ARACI
}

\begin{abstract}
We shall construct some new $p$-adic approaches to linear 2 -normed spaces by using the facts that known about $p$-adic numbers, $p$-adic analysis and give some results in this sense.
\end{abstract}

\section{INTRODUCTION}

Kummer, in 1850, first introduced to $p$-adic numbers. Then the German Mathematician, Kurt Hensel (1861-1941) developed the p-adic numbers in a paper which was concerned with the development of algebraic numbers in power series, around the end of the nineteenth century, in 1897. Then $p$ - adic numbers were generalized to ordinals (or valuation) by Kürschak in 1913, and Minkowski (1884), Tate (1960), Kubota-Leopoldt (1964), Iwasawa, Serre, Mazur, Manin, Katz, and the others. There are numbers of all kinds such as rational, real, complex, $p$-adic, ...Hensel's p-adic's numbers are now widely used in many fields such as analysis, physics and computer science. The $p$-adic numbers are less well known than the others, but they play a fundamental role in number theory in other parts of mathematics. Although, they have penatrated several mathematical fields, among them, number theory, algebraic geometry, algebraic topology and analysis. These numbers are now well-established in mathematical world and used more and more by physicists as well. Over the last century $p$-adic numbers and $p$ adic analysis have come to play an important role in number theory. They have many applications in mathematics, for example: Representation theory, algebraic geometry, and modern number theory and many applications in mathematical physics since 1897, for example; String theory, QFT, quantum mechanics, dynamical systems, complex systems, etc. Recently, Branko Dragovich in his study ([5]) he constructed $p$-adic approach to the genetic code and the genome and gave a new approach between $p$-adic fields and biology with chemistry, especially orghanic cemistry. The other researchers gave the different approach with $p$-adic on various disciplines of mathematics

2000 Mathematics Subject Classification. Primary: 11B68, 11F80, 46A15, 11S80, $41 \mathrm{~A} 65$.

Key words and phrases. 2-normed spaces, $p$-adic numbers, $p$-adic norm, $p$-adic 2-norm. 
and its allied subjects. But, up to now, no work on linear 2-normed spaces has been done in the sense of $p$-adic.

The concept of linear 2-normed spaces has been investigated by Gähler in $1965([9])$ and has been developed extensively in different subjects by others. Lewandowska published a series of papers on 2-normed sets and generalized 2-normed spaces in 1999-2003 ([15]-[17]).

In this paper we will not give a detailed information about $p$-adic number fields but we shall start with a review of $p$-adic numbers ( see ([1], [2], [3], [7], [8], [10], [11], [12], [13], [14], [19]) for more details) and 2-normed spaces and related concepts such as generalized 2-normed spaces, convergent sequences, 2-Banach spaces, ,..etc. (see ([4], [6],[9], [15], [16], [17], [20]) for more details.)

It is the aim of this paper is to give the reader a very understandable and readable connection between the concepts in $p$-adic numbers, $p$-adic analysis and linear 2-normed spaces. We shall not go deeper into the topic of $p$-adic analysis in our this first manuscript, but we hope in the next.

\section{Preliminaries}

Throughout this paper, we will use the notations; $p$ for a prime number, $\mathbb{Z}$ - the ring of rational integers, $\mathbb{Z}^{+}$- the positive integers, $\mathbb{Q}$ - the field of rational numbers, $\mathbb{R}$ - the field of real numbers, $\mathbb{R}^{+}$- the positive real numbers, $\mathbb{Z}_{p}$ - the ring of $p$-adic rational integers, $\mathbb{Q}_{p}$ - the field of $p$-adic rational numbers, $\mathbb{C}$ - the field of complex numbers and $\mathbb{C}_{p}$ - the $p$-adic completion of the algebraic closure of $\mathbb{Q}_{p}$. For each $x \in \mathbb{R}$, the absolute value of $x$ is denoted by $|x|$ and defined

$$
|x|=\left\{\begin{array}{cl}
-x & , \text { if } x \geq 0 \\
x & , \text { if } x \leq 0
\end{array} .\right.
$$

Thus $|0|=0$ and $|x|>0$ if $x \neq 0$. It is not difficult to check that $|x+y| \leq$ $|x|+|y|$ and $|x y| \leq|x||y|$ for every $x, y \in \mathbb{R}$, as usual.

In [21], some inequalities are defined as follows:

Suppose that $N(x)$, a non-negative real valued function defined on $\mathbb{Q}$ such that $N(0)=0, N(x)$ is positive if $x \neq 0$, and $N(x y)=N(x) N(y)$ for all $x, y \in \mathbb{Q}$, and

$$
N(x+y) \leq K(N(x)+N(y))
$$

for $K \geq 1$ and $x, y \in \mathbb{Q}$. The well-known triangle inequality satisfies for $K=1$. The other version of the triangle inequality, is the ultrametric which is stronger and is shown by

$$
N(x+y) \leq \max \{N(x), N(y)\}
$$

for all $x, y \in \mathbb{Q}$. By using the property $(2.1)$, we have

$$
N\left(\sum_{k=1}^{2^{n}} x_{k}\right) \leq K^{n} \sum_{k=1}^{2^{n}} N\left(x_{k}\right)
$$


where $n \in \mathbb{Z}^{+}$and $x_{k}$ 's are in $\mathbb{Q}$. The proof can be easily made by induction over $n$. The usual absolute value function, $|x|$ satisfies these conditions with the well-known triangle inequality. For $x=0$ and $x \neq 0$, if we have $N(x)=0$ and $N(x)=1$, respectively. In this case, $N(x)$ satisfies these conditions with the ultrametric type of the triangle inequality.

If $q \in \mathbb{C}$ we assume that $|q|<1$. If $q \in \mathbb{C}_{p}$, it will be assumed that $N(1-q)_{p}<p^{-\frac{1}{p-1}}$ with $N(p)_{p}<p^{-\operatorname{ord}_{p}(p)}=p^{-1}$, where $\operatorname{ord}_{p}(p)$ be the normalized exponential valuation of $\mathbb{C}_{p}$. We use the function

$$
[x]=[x: q]=\frac{1-q^{x}}{1-q}
$$

and

$$
\lim _{q \rightarrow 1} \frac{1-q^{x}}{1-q}=x
$$

for any $x$ in the complex case and any $x$ with $N(x)_{p} \leq 1$ in the $p$-adic case.

Every $x \in \mathbb{Q}$ with $|x|_{p} \leq 1$ is the limit of a sequence of integers in the $p$-adic metric. That is;

$$
\left\{x \in \mathbb{Q}:|x|_{p} \leq 1\right\}
$$

is the same as the closure of $\mathbb{Z}$ in $\mathbb{Q}$ with respect to the $p$-adic metric. Set

$$
\mathbb{Z}_{p}=\left\{x \in \mathbb{Q}_{p}:|x|_{p} \leq 1\right\} .
$$

Every $x \in \mathbb{Q}_{p}$ is the limit of a sequence of rational numbers in the $p$-adic metric. Because $\overline{\mathbb{Q}}=\mathbb{Q}_{p} \cdot(\overline{\mathbb{Q}}$ is the closure of $\mathbb{Q})$. It is also that for $x \in \mathbb{Z}_{p}$ in the $p$-adic metric. So we have, $\overline{\mathbb{Z}}=\mathbb{Z}_{p}$ in $\mathbb{Q}_{p}$ ( see [21] for details).

Now, let us give a brief knowledge about linear 2-normed spaces by starting their definitions and related facts with some examples.

Definition 1. Let $X$ be a linear space of dimension greater than 1 over $K$, where $K$ is the real or complex numbers field. Suppose $N(.,$.$) be a non-$ negative real-valued function on $X \times X$ satisfying the following conditions:

$(2 N 1) N(x, y)>0$ and $N(x, y)=0$ if and only if $x$ and $y$ are linearly dependent vectors,

(2N2) $N(x, y)=N(y, x)$ for all $x, y \in X$,

(2N3) $N(\lambda x, y)=|\lambda| N(x, y)$ for all $\lambda \in K$ and all $x, y \in X$,

$(2 N 4) N(x+y, z) \leq N(x, z)+N(y, z)$ for all $x, y, z \in X$.

Then $N(.,$.$) is called a 2-norm on X$ and the pair $(X, N(.,)$.$) is called a$ linear 2-normed space. In addition, for all scalars $\alpha$ and all $x, y, z \in X$, we have the following three properties of 2-norms:

$(P 1)$ They are non negative,

$(P 2) N(x, y)=N(x, y+\alpha x)$,

(P3) $N(x-z, y-z)=N(x-y, x-z)$. 
Every 2-normed space is a locally convex topological vector space. In fact for a fixed $b \in X, p_{b}(x)=N(x, b)$ for all $x \in X$, is a seminorm and the family $P=\left\{p_{b}: b \in X\right\}$ generates a locally convex topology on $X$. Such a topology is called the natural topology induced by 2-norm $N(.,$.$) .$

First we give some examples on linear 2-normed spaces.

Example 1. Let $X=\mathbb{R}^{3}$. Define

$N(x, y)=\max \left\{\left|x_{1} y_{2}-x_{2} y_{1}\right|+\left|x_{1} y_{3}-x_{3} y_{1}\right|,\left|x_{1} y_{2}-x_{2} y_{1}\right|+\left|x_{2} y_{3}-x_{3} y_{2}\right|\right\}$, where $x=\left(x_{1}, x_{2}, x_{3}\right)$ and $y=\left(y_{1}, y_{2}, y_{3}\right) \in \mathbb{R}^{3}$.

Then $N(.,$.$) is a 2$-norm on $\mathbb{R}^{3}$ (see [6] for details).

Example 2. Let $P_{n}$ denotes the set of real polynomials of degree less than or equal to $n$, on the interval $[0,1]$. By considering usual addition and scalar multiplication, $P_{n}$ is a linear vector space over the reals. Let $\left\{x_{1}, x_{2}, \ldots, x_{2 n}\right\}$ be distinct fixed points in $[0,1]$ and define the 2-norm on $P_{n}$ as

$$
N(f, g)=\sum_{k=1}^{2 n}\left|f\left(x_{k}\right) \cdot g^{\prime}\left(x_{k}\right)-f^{\prime}\left(x_{k}\right) \cdot g\left(x_{k}\right)\right| \text {. }
$$

Then $\left(P_{n}, N(.,).\right)$ is a 2-normed space.

Example 3. Let $X=\mathbb{R}^{3}$ and consider the following 2-norm on $X$ :

$$
N(x, y)=|x \times y|=\left|\operatorname{det}\left(\begin{array}{ccc}
i & j & k \\
x_{1} & x_{2} & x_{3} \\
y_{1} & y_{2} & y_{3}
\end{array}\right)\right|,
$$

where $x=\left(x_{1}, x_{2}, x_{3}\right)$ and $y=\left(y_{1}, y_{2}, y_{3}\right)$.Then $(X, N(.,)$.$) is a 2-normed$ space.

Definition 2. (i) A sequence $\left\{x_{n}\right\}_{n \geq 1}$ in a 2-normed space $(X, N(.,)$.$) is$ called a Cauchy sequence if there exist two linearly independent elements $y$ and $z$ in $X$ such that $\left\{N\left(x_{n}, y\right)\right\}$ and $\left\{N\left(x_{n}, z\right)\right\}$ are real Cauchy sequences.

(ii) A sequence $\left\{x_{n}\right\}_{n>1}$ in a 2-normed space $(X, N(.,)$.$) is called conver-$ gent if there exists $x \in \bar{X}$ such that $\left\{N\left(x_{n}-x, y\right)\right\}_{n \geq 1}$ thends to zero for all $y \in X$.

(iii) A 2-normed space $(X, N(.,)$.$) is called 2-Banach space if every$ Cauchy sequence is convergent.

Lemma 1. [6] (i) Every 2-normed space of dimension 2 is a 2-Banach space, when the underlying field is complete.

(ii) If $\left\{x_{n}\right\}_{n \geq 1}$ is a sequence in 2-normed space $(X, N(.,)$.$) and$ $\lim _{n \rightarrow \infty} N\left(x_{n}-x, y\right)=0$, then $\lim _{n \rightarrow \infty} N\left(x_{n}, y\right)=N(x, y)$. 
3. P-ADIC ORDINAL, P-ADIC NORM, P-ADIC METRIC, P-ADIC EXPANSION AND RELATED EXAMPLES

In this section, we introduce the notions of $p$-adic ordinal, $p$-adic norm, $p$-adic metric(distance), $p$-adic expansion and some related concepts with some examples. Let $\mathbb{Q}$ be the field of rational numbers, $0 \neq x \in \mathbb{Q}$ and $p$ is a fixed prime number. Every rational number can be represented in the form

$$
x=p^{n} \cdot \frac{a}{b}
$$

with $\operatorname{gcd}(a, b)=1, a, n \in \mathbb{Z}, b \in \mathbb{Z}^{+}$and neither $a$ nor $b$ is divisible by $p$ (that is $(p, a)=1,(p, b)=1$.). The integer $n$ and the rational number $\frac{a}{b}$ are well defined by F.T.A. (Fundamental Theorem of Arithmetic). Now, let us give the definition of $p$-adic ordinal as follows:

Definition 3. The $p$-adic ordinal (or valuation) is the function

$$
\operatorname{ord}_{p}: \mathbb{Q} \rightarrow \mathbb{Z} \cup\{\infty\}
$$

with

$$
\operatorname{ord}_{p}(x)=n \text { for } 0 \neq x \in \mathbb{Q} \text { and } \operatorname{ord}_{p}(0)=\infty .
$$

For all $x, y \in \mathbb{Q}$, we have the following some basic facts:

1) $\operatorname{ord}_{p}(x y)=\operatorname{ord}_{p}(x)+\operatorname{ord}_{p}(y)$,

2) $\operatorname{ord}_{p}(x+y) \geq \min \left\{\operatorname{ord}_{p}(x), \operatorname{ord}_{p}(y)\right\}$, with equality when $\operatorname{ord}_{p}(x) \neq$ $\operatorname{ord}_{p}(y)$

3) $\operatorname{ord}_{p}(0)=\infty$,

4) A clear consequence of the first property is that $\operatorname{ord}_{p}\left(\frac{x}{y}\right)=$ $\operatorname{ord}_{p}(x)-\operatorname{ord}_{p}(y)$.

Example 4. Let us suppose $p=3$. Then we have:

$$
\operatorname{ord}_{3}(3)=\operatorname{ord}_{3}\left(3^{1} .1\right)=1, \operatorname{ord}_{3}(5)=\operatorname{ord}_{3}\left(5^{1} \cdot 3^{0}\right)=0,
$$

and

$$
\operatorname{ord}_{3}\left(\frac{1}{9}\right)=\operatorname{ord}_{3}\left(3^{-2} \cdot 1\right)=-2 .
$$

By using the ordinal(valuation) function, we can define an $p$-adic norm function on $\mathbb{Q}$, as follows:

Definition 4. For $x \in \mathbb{Q}$, let the $p$-adic norm of $x$ be given by

$$
N(x)_{p}=\left\{\begin{array}{cl}
p^{-\operatorname{ord}_{p} x} & , \text { for } x \neq 0 \\
p^{-\infty}=0 & , \text { for } x=0
\end{array} .\right.
$$


Example 5. Let $x=\frac{63}{550}=\frac{7 \cdot 3^{2}}{2 \cdot 5^{2} \cdot 11}=2^{-1} \cdot 3^{2} \cdot 5^{-2} \cdot 7^{1} \cdot 11^{-1}$. Then we have the followings.

$$
\begin{aligned}
& N\left(\frac{63}{550}\right)_{p=2}=2, N\left(\frac{63}{550}\right)_{p=3}=\frac{1}{9}, N\left(\frac{63}{550}\right)_{p=5}=25, \\
& N\left(\frac{63}{550}\right)_{p=7}=\frac{1}{7}, N\left(\frac{63}{550}\right)_{p=11}=11
\end{aligned}
$$

and for the others $N\left(\frac{63}{550}\right)_{p \geq 13}=1$.

The $p$-adic norm satisfies the following relations:

(i) $N(x)_{p} \geq 0$ for all $x$,

(ii) $N(x)_{p}=0$ if and only if $x=0$,

(iii) $N(x y)_{p}=N(x)_{p} \cdot N(y)_{p}$ for all $x$, and $y$,

(iv) $N(x+y)_{p} \leq N(x)_{p}+N(y)_{p}$ for all $x$, and $y$,

(v) $N(x+y)_{p} \leq \max \left\{N(x)_{p}, N(y)_{p}\right\}$ for all $x$, and $y$.

$$
\begin{aligned}
(v(i)) & \text { if } N(x)_{p} \neq N(y)_{p} \text { then } N(x-y)_{p}=\max \left\{N(x)_{p}, N(y)_{p}\right\}, \\
(v(i i)) & \text { if } N(x)_{p}=N(y)_{p} \text { then } N(x-y)_{p}=N(x)_{p} .
\end{aligned}
$$

In the above, the properties, $(i v)$ and $(v)$ are called the triangle inequality and the strong triangle inequality (ultrametric version of the triangle inequality) respectively. We observe that the relation $(i v)$ follows from the relation $(v)$.

By

$$
d_{p}(x, y)=N(x-y)_{p}
$$

we define the $p$-adic metric (distance) on $\mathbb{Q}$, for fixed a prime number $p$, and $x, y \in \mathbb{Q}$, as follows:

Definition 5. For all $x, y, z \in \mathbb{Q}$,

D1) $d_{p}(x, y)=N(x-y)_{p}>0$ for $x \neq y$ and $d_{p}(x, x)=0$,

D2) $d_{p}(x, y)=d_{p}(y, x)$

D3) $d_{p}(x, z) \leq d_{p}(x, y)+d_{p}(y, z)$, ( the triangle inequality),

D4) $d_{p}(x, z) \leq \max \left\{d_{p}(x, y), d_{p}(y, z)\right\}$, ( the ultrametric inequality).

The properties given above from 1 to 3 , they are called the axioms of $p$-adic metric and the pair $\left(X, d_{p}\right)$ is called a $p$-adic metric space. If the metric also satisfies the fourth property then this metric is called a $p$-adic ultrametric space.

To prove from (D1) to (D4); For all $x, y, z \in \mathbb{Q}$, we suppose that $x \neq y$. For $x-y$, we have

$$
x-y=p^{n} \frac{a}{b} \text { for } n \in \mathbb{Z}, \quad \frac{a}{b} \in \mathbb{Q} \text { and } \operatorname{ord}_{p}\left(\frac{a}{b}\right)=0,
$$

where $\operatorname{gcd}(a, b)=1$ and neither $a$ nor $b$ is divisible by $p$. 
Firstly,

$$
d_{p}(x, y)=\left|p^{n} \cdot \frac{a}{b}\right|_{p}=p^{-n}=\frac{1}{p^{n}}>0 .
$$

Also, by definition, we obtain

$$
d_{p}(x, x)=|x-x|_{p}=|0|_{p}=0
$$

and

$$
d_{p}(x, y)=|x-y|_{p}=p^{-\operatorname{ord}_{p}(x-y)}=p^{-\operatorname{ord}_{p}(y-x)}=|y-x|_{p}=d_{p}(y, x) .
$$

Now, let us prove the property (D4); For this we have

$$
\begin{aligned}
d_{p}(x, z) & =|x-z|_{p}=|(x-y)-(z-y)|_{p} \\
& =p^{-\operatorname{ord}_{p}((x-y)-(z-y))} \\
& \leq p^{-\min \left(\operatorname{ord}_{p}(x-y), \operatorname{ord}_{p}(z-y)\right)} \\
& =\max \left\{d_{p}(x, y), d(z, y)\right\} \\
& =\max \left\{d_{p}(x, y), d_{p}(y, z)\right\} .
\end{aligned}
$$

The property (D4) implies property (D3). So the desired proof completes.

Two points are $p$-adically closer as long as $r$ is higher, such that $p^{r}$ divides $N(x-y)_{p}$. Amazingly, for $p=5$, the result is that 135 is closer to 10 than 35 .

Example 6. For $p=7$-adic metric; we have

$$
d(3,52)<d(3,4)
$$

since

and

$$
d(3,52)=|52-3|_{p=7}=|49|_{p=7}=\left|7^{2}\right|_{p=7}=\frac{1}{7^{2}}=\frac{1}{49}
$$

$$
d(3,4)=|4-3|_{p=7}=|1|_{p=7}=\left|7^{0}\right|_{p=7}=\frac{1}{7^{0}}=1 .
$$

Definition 6. A sequence $\left(x_{n}\right)_{n=1}^{\infty}$ of rational numbers converges to $x \in \mathbb{Q}$ in $p$-adic metric if for every $\varepsilon>0$, there is an $l \geq 1$ such that

$$
d_{p}\left(x_{n}, x\right)=\left|x_{n}-x\right|_{p}<\varepsilon
$$

for every $n \geq l$.

For the given two sequences of rational numbers which are $\left(x_{n}\right)_{n=1}^{\infty}$ and $\left(y_{n}\right)_{n=1}^{\infty}$ converge to $x, y \in \mathbb{Q}$, in the $p$-adic metric respectively, then the sequence of sums $x_{n}+y_{n}$ and product $x_{n} y_{n}$ converge to the sum, $x+y$ and to the product, $x y$ of the limits of initial sequences.

Definition 7. A sequence $\left(x_{n}\right)_{n=1}^{\infty}$ of rational numbers is a Cauchy sequence with respect to the $p$-adic metric if for each $\varepsilon>0$ there is an $l \geq 1$ such that

$$
d_{p}\left(x_{n}, x_{m}\right)=\left|x_{n}-x_{m}\right|_{p}<\varepsilon
$$

for all $n, m \geq l$. 
Every convergent sequence in $\mathbb{Q}$ is a Cauchy sequence. If $\left(x_{n}\right)_{n=1}^{\infty}$ is a Cauchy sequence in $\mathbb{Q}$ with respect to the $p$-adic metric, then the limit

$$
\lim _{n \rightarrow \infty}\left(x_{n}-x_{n+1}\right)=0
$$

in $p$-adic metric. We know that the analogous statement also works for the standard metric $|x-y|$. For the $p$-adic metric, the converse holds because of the ultrametric version of the triangle inequality.

Definition 8. ([13]) A p-adic number $\alpha$ can be uniquely written in the canonical series form

$$
\alpha=\sum_{j=n}^{\infty} a_{j} p^{j}
$$

where each of $0 \leq a_{j} \leq p-1$ and the $p$-adic norm of the number $\alpha$ is defined as $N(\alpha)_{p}=p^{-n}$. Note that the series

$$
1+p+p^{2}+p^{3}+\cdots
$$

converges to $\frac{1}{1-p}$ in the $p$-adic norm. Now,let us consider the power series expansion, as an example;

$$
\begin{aligned}
\alpha & =2+3 p+p^{2}+3 p^{3}+p^{4}+3 p^{5}+p^{6}+\cdots \\
& =2+3 p\left(1+p^{2}+p^{4}+\cdots\right)+p^{2}\left(1+p^{2}+p^{4}+\cdots\right) \\
& =2+\left(3 p+p^{2}\right)\left(1+p^{2}+p^{4}+\cdots\right)
\end{aligned}
$$

since $1+p^{2}+p^{4}+\cdots$ converges to $\left(1-p^{2}\right)^{-1}$, we have

$$
\alpha=2+\frac{3 p+p^{2}}{1-p^{2}} .
$$

For $p=5$, we have 5 -adic expansion of $\alpha=\frac{1}{3}$, which can be written in the form

$$
\begin{aligned}
\frac{1}{3} & =0.2313131 \cdots \quad(p=5) \\
& =0.2 \overline{31} \quad(p=5) .
\end{aligned}
$$

The purpose of this paper is to construct a new $p$-adic approach to linear 2 -normed spaces by using the facts that known about $p$-adic numbers and $p$-adic analysis.

\section{P-Adic Approach to Linear 2-Normed Spaces}

The main results of this paper are given in this section with new definitions we have done in the sense of linear 2-normed spaces. Here we present a $p$ adic version of the results of linear 2-normed spaces and we will investigate some elementary $p$-adic analysis, including concepts such as convergence of sequences, completion of sequences, Banach spaces and other concepts (topics) familiar from elementary real analysis, in the field of the $p$-adic 
numbers, $\mathbb{Q}_{p}$ with the $p$-adic norm $N(.)_{p}$, but now in the context of $p$-adic linear 2-normed spaces over $\mathbb{Q}_{p}$ with the $p$-adic 2-norm $N(.,)_{p}$.

Definition 9. (i) The $p$-adic ordinal (valuation) of $x$, and $y$, for $0 \neq x, y \in$ $\mathbb{Z}$, is

$$
\operatorname{ord}_{p}(x, y)=\max \left\{r: p^{r} \mid x \text { and } p^{r} \mid y\right\} \geq 0 .
$$

(ii) For $\frac{a}{b}, \frac{c}{d} \in Q$, The p-adic value of $\frac{a}{b}$ and $\frac{c}{d}$ is

$$
\operatorname{ord}_{p}\left(\frac{a}{b}, \frac{c}{d}\right)=\operatorname{ord}_{p}(a, c)-\operatorname{ord}_{p}(a, d)-\operatorname{ord}_{p}(b, c)+\operatorname{ord}_{p}(b, d) .
$$

(iii) For $\frac{a}{b}, c \in Q$, with $d=1$; The p-adic value of $\frac{a}{b}$ and $c$ is

$$
\operatorname{ord}_{p}\left(\frac{a}{b}, c\right)=\operatorname{ord}_{p}(a, c)-\operatorname{ord}_{p}(b, c) .
$$

Notice that in all cases, $\operatorname{ord}_{p}$, in 2-norm, gives an integer and that for rational numbers $\frac{a}{b}$, and $\frac{c}{d}$ the value of $\operatorname{ord}_{p}\left(\frac{a}{b}, \frac{c}{d}\right)$ is well defined. i.e., if

$$
\frac{a}{b}=\frac{a^{\prime}}{b^{\prime}} \text { and } \frac{c}{d}=\frac{c^{\prime}}{d^{\prime}}
$$

then

$$
\operatorname{ord}_{p}\left(\frac{a}{b}, \frac{c}{d}\right)=\operatorname{ord}_{p}\left(\frac{a^{\prime}}{b^{\prime}}, \frac{c^{\prime}}{d^{\prime}}\right)
$$

We also introduce the convention that $\operatorname{ord}_{p}(0, y)=\operatorname{ord}_{p}(x, 0)=\infty$.

For example; by taking

$$
\frac{a}{b}=\frac{1}{7}, \frac{c}{d}=\frac{1}{5}, \frac{a^{\prime}}{b^{\prime}}=\frac{3}{21}, \frac{c^{\prime}}{d^{\prime}}=\frac{3}{15}
$$

and for $p=7$, using the definition 9 , we have

$$
\begin{aligned}
\operatorname{ord}_{7}\left(\frac{1}{7}, \frac{1}{5}\right) & =\operatorname{ord}_{7}(1,1)-\operatorname{ord}_{7}(1,5)-\operatorname{ord}_{7}(7,1)+\operatorname{ord}_{7}(7,5) \\
& =0
\end{aligned}
$$

and

$$
\begin{aligned}
\operatorname{ord}_{7}\left(\frac{3}{21}, \frac{3}{15}\right) & =\operatorname{ord}_{7}(3,3)-\operatorname{ord}_{7}(3,15)-\operatorname{ord}_{7}(21,3)+\operatorname{ord}_{7}(21,15) \\
& =0
\end{aligned}
$$

For example; by taking

$$
\frac{a}{b}=\frac{2}{5}, \frac{c}{d}=\frac{3}{5}, \frac{a^{\prime}}{b^{\prime}}=\frac{4}{10}, \frac{c^{\prime}}{d^{\prime}}=\frac{6}{10}
$$

and for $p=5$, using the definition 9, we have

$$
\begin{aligned}
\operatorname{ord}_{5}\left(\frac{2}{5}, \frac{3}{5}\right) & =\operatorname{ord}_{5}(2,3)-\operatorname{ord}_{5}(2,5)-\operatorname{ord}_{5}(5,3)+\operatorname{ord}_{5}(5,5) \\
& =1
\end{aligned}
$$


and

$$
\begin{aligned}
\operatorname{ord}_{5}\left(\frac{4}{10}, \frac{6}{10}\right) & =\operatorname{ord}_{5}(4,6)-\operatorname{ord}_{5}(4,10)-\operatorname{ord}_{5}(10,6)+\operatorname{ord}_{5}(10,10) \\
& =1
\end{aligned}
$$

For example; by taking

$$
\frac{a}{b}=\frac{2}{4}, \frac{c}{d}=\frac{4}{16}, \frac{a^{\prime}}{b^{\prime}}=\frac{6}{12}, \frac{c^{\prime}}{d^{\prime}}=\frac{12}{48}
$$

and for $p=2$, using the definition 9 , we have

$$
\begin{aligned}
\operatorname{ord}_{2}\left(\frac{2}{4}, \frac{4}{16}\right) & =\operatorname{ord}_{2}(2,4)-\operatorname{ord}_{2}(4,4)-\operatorname{ord}_{2}(2,16)+\operatorname{ord}_{2}(4,16) \\
& =0
\end{aligned}
$$

and

$$
\begin{aligned}
\operatorname{ord}_{2}\left(\frac{6}{12}, \frac{12}{48}\right) & =\operatorname{ord}_{2}(6,12)-\operatorname{ord}_{2}(12,12)-\operatorname{ord}_{2}(6,48)+\operatorname{ord}_{2}(12,48) \\
& =0
\end{aligned}
$$

Example 7. For $\frac{7}{3}, 4 \in Q$, The $p=5$-adic value of $\frac{7}{3}$ and 4 is

$$
\begin{aligned}
\operatorname{ord}_{5}\left(\frac{7}{3}, 4\right)= & \operatorname{ord}_{5}(7,4)-\operatorname{ord}_{5}(3,4) \\
= & \max \left\{r: 5^{r} \mid 7 \text { and } 5^{r} \mid 4\right\}- \\
& -\max \left\{r: 5^{r} \mid 3 \text { and } 5^{r} \mid 4\right\} \\
= & 0
\end{aligned}
$$

and

$$
\begin{aligned}
\operatorname{ord}_{5}\left(\frac{25}{3}, 5\right)= & \operatorname{ord}_{5}(25,5)-\operatorname{ord}_{5}(3,5) \\
= & \max \left\{r: 5^{r} \mid 25 \text { and } 5^{r} \mid 5\right\}- \\
& -\max \left\{r: 5^{r} \mid 3 \text { and } 5^{r} \mid 5\right\} \\
= & 1-0 \\
= & 1
\end{aligned}
$$

The $p$-adic valuation has the following properties:

Proposition 1. For all $x, y \in \mathbb{Q}$, we have for $\operatorname{ord}_{p}$;

(i) $\operatorname{ord}_{p}(x, y)=\infty$ iff $x=0$, or $y=0$,

(ii) $\operatorname{ord}_{p}(x z, y)=\operatorname{ord}_{p}(x, y)+\operatorname{ord}_{p}(z, y)$,

(iii) $\operatorname{ord}_{p}(x+z, y) \geq \min \left\{\operatorname{ord}_{p}(x, y), \operatorname{ord}_{p}(z, y)\right\}$ implies $\operatorname{ord}_{p}(x, y) \neq \operatorname{ord}_{p}(z, y)$. 
Proof. (i) $\operatorname{ord}_{p}(x, y)=\max \left\{r: p^{r}\left|x, p^{r}\right| y\right\}=\infty$. We know that $\operatorname{ord}_{p}(0, y)=\operatorname{ord}_{p}(x, 0)=\infty$.

(ii)

$$
\begin{aligned}
\operatorname{ord}_{p}(x z, y) & =\max \left\{r: p^{r}\left|x z, p^{r}\right| y\right\} \\
& =\max \left\{r:\left(p^{r} \mid x \text { and } p^{r} \mid y\right) \text { and }\left(p^{r} \mid z \text { and } p^{r} \mid y\right)\right\} \\
& =\max \left\{r: p^{r}\left|x, p^{r}\right| y\right\}+\max \left\{r: p^{r}\left|z, p^{r}\right| y\right\} \\
& =\operatorname{ord}_{p}(x, y)+\operatorname{ord}_{p}(z, y) .
\end{aligned}
$$

(iii) Let $x, y, z$ be non zero elements of rational numbers. Write $x=p^{r} \frac{a}{b}$ and $z=p^{s} \frac{c}{d}$, where $a, b, c, d \in \mathbb{Z}$ with $p \nmid a, b, c, d$ and $r, s \in \mathbb{Z}$. Now, if $r=s$, we have

$$
\begin{aligned}
x+z & =p^{r}\left(\frac{a}{b}+\frac{c}{d}\right) \\
& =p^{r}\left(\frac{a d+b c}{b d}\right)
\end{aligned}
$$

which gives $\operatorname{ord}_{p}(x+z, y) \geq r$ since $p \nmid b d$. Now suppose that $r \neq s$, and say $s>r$.

$$
\begin{aligned}
x+z & =p^{r}\left(\frac{a}{b}+p^{s-r} \frac{c}{d}\right) \\
& =p^{r}\left(\frac{a d+p^{s-r} b c}{b d}\right)
\end{aligned}
$$

Notice that $s-r>0$ and $p \nmid a d$, then, we have

$$
\operatorname{ord}_{p}(x+z, y) \geq \min \left\{\operatorname{ord}_{p}(x, y), \operatorname{ord}_{p}(z, y)\right\} .
$$

Now, we consider our fundamental definition of this paper.

Definition 10. For $x, y \in \mathbb{Q}$, let the $p$-adic norm of $x, y$ be given by

$$
N(x, y)_{p}=\left\{\begin{array}{cc}
p^{-\operatorname{ord}_{p}(x, y)} & , \quad \text { for } x, y \neq 0 \\
p^{-\infty}=0 & , \quad \text { for } x=0 \text { or } y=0
\end{array}\right.
$$

where

$$
\operatorname{ord}_{p}(x, y)=\max \left\{r: p^{r} \mid x \text { and } p^{r} \mid y\right\} .
$$

Example 8. Let $x=\frac{2}{5}$ and $y=\frac{3}{5}$. Using the above definition, we have the followings:

$$
N(x, y)_{p}=N\left(\frac{2}{5}, \frac{3}{5}\right)_{p=5}=\frac{1}{5},
$$

since $\operatorname{ord}_{5}\left(\frac{2}{5}, \frac{3}{5}\right)=1$ from example given above.

Proposition 2. Let the function $N(., .)_{p}$ be a non-negative real-valued function on $Q \times Q$ satisfying the following conditions:

$$
N(., .)_{p}: Q \times Q \rightarrow R^{+} \cup\{0\}=\{r: r \geq 0\}
$$


(a) $N(x, z)_{p}=0$ if and only if $x=0$ or $z=0$,

(a) $N(x y, z)_{p}=N(x, z)_{p} \cdot N(y, z)_{p}$ for all $x, y$ and $z \in \mathbb{Q}$,

(c). $N(x+y, z)_{p} \leq \max \left\{N(x, z)_{p}, N(y, z)_{p}\right\}$ and with equality $N(x, z)_{p} \neq$ $N(y, z)_{p}$,

where $N(., .)_{p}$ is a non-Archimedean norm on $Q$.

Proof. (i) $N(x, z)_{p}=0 \Rightarrow \operatorname{ord}_{p}(x, z)=\infty \Rightarrow x=0$ or $z=0$. If $x=0$ and $z=0$ then

$$
N(x, z)_{p}=p^{-\operatorname{ord}_{p}(0)}=p^{-\infty}=\frac{1}{p^{\infty}}=0 .
$$

(ii) $N(x y, z)_{p}=N(x, z)_{p} \cdot N(y, z)_{p}$

(a) If $x y=0$ or $z=0$ then $N(x y, z)_{p}=0$.

(b) If $x y \neq 0$ or $z \neq 0$ then

$$
\begin{aligned}
N(x y, z)_{p} & =p^{-\operatorname{ord}_{p}(x y, z)}=p^{-\operatorname{ord}_{p}(x, z)-\operatorname{ord}_{p}(y, z)} \\
& =p^{-\operatorname{ord}_{p}(x, z)} \cdot p^{-\operatorname{ord}_{p}(y, z)} \\
& =N(x, z)_{p} \cdot N(y, z)_{p} .
\end{aligned}
$$

(c) To prove this property, we have

$$
\begin{aligned}
N(x+y, z)_{p} & =p^{-\operatorname{ord}_{p}(x+y, z)}=\frac{1}{p^{\operatorname{ord}_{p}(x+y, z)}} \\
& \leq \frac{1}{p^{\min \left\{\operatorname{ord}_{p}(x, z), \operatorname{ord}_{p}(y, z)\right\}}} \\
& =p^{-\min \left\{\operatorname{ord}_{p}(x, z), \operatorname{ord}_{p}(y, z)\right\}} \\
& =\max \left\{p^{-\operatorname{ord}_{p}(x, z)}, p^{-\operatorname{ord}_{p}(y, z)}\right\} \\
& =\max \left\{N(x, z)_{p}, N(y, z)_{p}\right\} .
\end{aligned}
$$

This is the desired result.

Let $N(x, z)_{p}$ be a non-negative real valued function defined on the rational numbers $Q \times Q$ such that $N(x, z)_{p}=0$ for $x=0$ or $z=0, N(x, z)_{p}>0$ when $x \neq 0, z \neq 0 . N(x y, z)_{p}=N(x, z)_{p} \cdot N(y, z)_{p}$ for all $x, y, z \in \mathbb{Q}$ and

$$
N(x+y, z)_{p} \leq K\left(N(x, z)_{p}+N(y, z)\right)_{p}
$$

for some $K \geq 1$ and all $x, y, z \in \mathbb{Q}$. For the usual triangle inequality one ask that this condition holds with $K=1$, i.e.,

$$
N(x+y, z)_{p} \leq N(x, z)_{p}+N(y, z)_{p}
$$

for all $x, y, z \in \mathbb{Q}$. 
The ultrametric version of the triangle inequality is stronger still and asks that

$$
N(x+y, z)_{p} \leq \max \left(N(x, z)_{p}, N(y, z)_{p}\right)
$$

for all $x, y, z \in \mathbb{Q}$. If $N(.,$.$) satisfies (4.1), n$ is a positive integer and $x_{1}, x_{2}, x_{3}, \ldots, x_{2^{n}}, z \in \mathbb{Q}$, then

$$
N\left(\sum_{k=1}^{2^{n}} x_{k}, z\right)_{p} \leq K^{n} \sum_{k=1}^{2^{k}} N\left(x_{k}, z\right)_{p}
$$

as one can check using induction on $k$. For all $a>0, N(x, z)^{a}$ is a nonnegative real-valued function on $\mathbb{Q}$ which vanishes at 0 , is positive at all nonzero $x \in \mathbb{Q}$, and sends products to products.

If $N(x, z)_{p}$ satisfies $(4.1)$, then

$$
N(x+y, z)_{p}^{a} \leq K^{a}\left(N(x, z)_{p}^{a}+N(y, z)_{p}^{a}\right)
$$

when $0<a \leq 1$ and

$$
N(x+y, z)_{p}^{a} \leq 2^{a-1} \cdot K^{a}\left(N(x, z)_{p}^{a}+N(y, z)_{p}^{a}\right)
$$

when $a \geq 1$.

In particular, if $N(x, z)_{p}$ satisfies the well-known triangle inequality (4.2) and $0<a \leq 1$, then $N(x, z)_{p}^{a}$ also satisfies the well-known triangle inequality. If $N(x, z)_{p}$ satisfies the ultrametric version (4.3) of the triangle inequality, then $N(x, z)_{p}^{a}$ satisfies the ultrametric version of the triangle inequality for all $a \geq 0$.

Definition 11. Let $X$ be a linear space of dimension greater than 1 over $K$, where $K$ is the real or complex numbers field. Suppose $N(.,$.$) be a non-$ negative real-valued function on $X \times X$ satisfying the following conditions:

(2N1) $N(x, y)_{p}=0$ if and only if $x$ and $y$ are linearly dependent vectors.

(2N2) $N(x y, z)_{p}=N(x, z)_{p} . N(y, z)_{p}$ for all $x, y, z \in X$,

(2N3) $N(x+y, z)_{p} \leq N(x, z)_{p}+N(y, z)_{p}$ for all $x, y, z \in X$, (2N4) $N(\lambda x, y)_{p}=\lambda N(x, y)_{p}$ for all $\lambda \in K$ and all $x, y \in X$.

Then $N(., .)_{p}$ is called a $p$-adic 2 -norm on $X$ and the pair $\left(X, N(., .)_{p}\right)$ is called a $p$-adic linear 2-normed space.

Definition 12. A sequence $\left\{x_{n}\right\}_{n \geq 1}$ in a $p$-adic linear 2-normed space $\left(X, N(., .)_{p}\right)$ is called convergent if there exists an $x \in X$ such that

$$
\lim _{n \rightarrow \infty} N\left(x_{n}-x, z\right)_{p}=0
$$

for all $z \in X$. 
Definition 13. A sequence $\left\{x_{n}\right\}_{n \geq 1}$ in a $p$-adic linear 2-normed space $\left(X, N(.,)_{p}\right)$ is called a Cauchy sequence if, for $\forall \varepsilon>0$ there exists $\exists M_{1} \in$ $X, n, m>M_{1}$ such that $N\left(x_{m}-x_{n}, z\right)_{p}<\varepsilon$.

Definition 14. A $p$-adic linear 2-normed space $\left(X, N(., .)_{p}\right)$ is called a complete if every Cauchy sequence is convergent in $p$-adic linear 2-normed space.

Definition 15. A $p$-adic linear 2-normed space $\left(X, N(., .)_{p}\right)$ is called a $p$-adic 2 -Banach space if every $p$-adic linear 2-normed space is complete.

Proposition 3. If $\lim _{n \rightarrow \infty} N\left(x_{n}, z\right)_{p}$ exists then we say that $\left\{x_{n}\right\}$ is a Cauchy sequence with respect to $N(., .)_{p}$.

Proof. Let us suppose that $\lim _{n \rightarrow \infty} N\left(x_{n}, z\right)_{p}=x$. Then we can obtain a constant $M_{1}$ such that $n>M_{1} \Rightarrow N\left(x-x_{n}, z\right)_{p}<\frac{\varepsilon}{2}$. If $m, n>M_{1}$, then

$$
N\left(x-x_{n}, z\right)_{p}<\frac{\varepsilon}{2} \text { and } N\left(x-x_{m}, z\right)_{p}<\frac{\varepsilon}{2},
$$

hence by using the triangle inequality, we have

$$
\begin{aligned}
N\left(x_{m}-x_{n}, z\right)_{p} & =N\left(x_{m}-x+x-x_{n}, z\right)_{p} \\
& \leq N\left(x_{m}-x, z\right)_{p}+N\left(x-x_{n}, z\right)_{p} \\
& <\frac{\varepsilon}{2}+\frac{\varepsilon}{2}=\varepsilon . \quad \square
\end{aligned}
$$

Definition 16. A sequence $\left(x_{n}\right)$ is called a null sequence in $p$-adic linear 2-normed space, if

$$
\lim _{n \rightarrow \infty} N\left(x_{n}, x\right)_{p}=0 .
$$

Example 9. Let $x_{n}=p^{n}$ and $z=p^{r}$ with $r<n$ in the $p$-adic 2-norm over $X=\mathbb{Q}$. Then

$$
N\left(p^{n}, p^{r}\right)_{p}=\left\{\begin{array}{cc}
p^{-\operatorname{ord}_{p}\left(p^{n}, p^{r}\right)} & , \text { if } p^{n} \neq 0 \text { and } p^{r} \neq 0 \\
p^{-\infty} & , \quad \text { if } p^{n}=0 \text { or } p^{r}=0 .
\end{array} .\right.
$$

In this case $N\left(p^{n}, p^{r}\right)_{p}=p^{-n}=\frac{1}{p^{n}}=0$, as $n \rightarrow \infty$. Therefore, $\lim _{n \rightarrow \infty} N\left(x_{n}, x\right)_{p}=0$. Hence this sequence is a null sequence with respect to the $p$-adic 2-norm.

Definition 17. A $p$-adic number $(\alpha, \beta)$ can be uniquely written in the form

$$
(\alpha, \beta)=\sum_{i=n, j=m}^{\infty}\left(a_{i} p^{i}, b_{j} p^{j}\right)
$$

where each of $0 \leq a_{i}, b_{j} \leq p-1$ and $p$-adic 2 -norm of the number $(\alpha, \beta)$ is defined as $N(\alpha, \beta)_{p}=n,(n \in R)$ and the double series

$$
\left(1+p+p^{2}+p^{3}+\cdots, 1+p+p^{2}+p^{3}+\cdots\right)
$$


converges to $\frac{1}{1-p}$ in the $p$-adic 2 -norm.

Example 10. Let $\alpha=4.13131313 \cdots$ and $\beta=0.02313131 \cdots$ with $p=5$. Set $(\alpha, \beta)$ as follows, we have

$$
\begin{aligned}
(\alpha, \beta)= & (4.13131313 \ldots, 0.02313131 \ldots) \\
= & \left(4.5^{-1}+1.5^{0}+3.5^{1}+1.5^{2}+3.5^{3}+\ldots,\right. \\
& \left.0.5^{0}+2.5^{1}+3.5^{2}+1.5^{3}+\ldots\right) \\
= & \left(\frac{4}{5}+\left(1+5^{2}+5^{4}+\ldots\right)+3.5\left(1+5^{2}+5^{4}+\ldots\right),\right. \\
& \left.5+5\left(1+5^{2}+5^{4}+\ldots\right)+3.5^{2}\left(1+5^{2}+5^{4}+\ldots\right)\right) \\
= & \left(\frac{4}{5}+16\left(1+5^{2}+5^{4}+\ldots\right), 5+80\left(1+5^{2}+5^{4}+\ldots\right)\right) \\
= & \left(\frac{4}{5}+16\left(\frac{1}{1-25}\right), 5+80\left(\frac{1}{1-25}\right)\right) \\
= & \left(4-\frac{2}{3}, 5-\frac{10}{3}\right) \\
= & \left(\frac{2}{15}, \frac{5}{3}\right)
\end{aligned}
$$

then

$$
\begin{aligned}
\operatorname{ord}_{5}\left(\frac{2}{15}, \frac{5}{3}\right) & =\operatorname{ord}_{5}(2,5)-\operatorname{ord}_{5}(2,3)-\operatorname{ord}_{5}(15,5)+\operatorname{ord}_{5}(15,3) \\
& =-1 .
\end{aligned}
$$

Finally, we obtain

$$
N\left(\frac{2}{15}, \frac{5}{3}\right)_{5}=5^{-\operatorname{ord}_{5}\left(\frac{2}{15}, \frac{5}{3}\right)}=5^{-(-1)}=5 .
$$

\section{REFERENCES}

[1] G. Bachman, Introduction to $p$-adic numbers and valuation theory, Academic press, 1964.

[2] G. Bachman, L. Narici, Functional Analysis, Academic Press, New York and London.

[3] A.J. Baker, An introduction to $p$-adic numbers and $p$-adic analysis, URL: http:// www.maths.gla.ac.uk/ ajb.

[4] Y. Cho, P. Lin, S.S. Kim, A. Misiak, Theory of 2-Inner Product Spaces, Nova Science Publishers, 2001.

[5] B. Dragovich, p-adic approach to the genetic code and genome, Institute of Physics, Belgrade, Serbia, TAG, 20-24 Oct, 2008, Annecy.

[6] R. Freese, Y. Cho, Geometry of Linear 2-Normed Spaces, Nova Science Publishers, 2001.

[7] F.Q. Gouvea, p-adic Numbers: An introductory survey, Berlin: Springer-Verlag.

[8] F.Q. Gouvea, p-adic: An introduction, universitext, Springer-Verlag, 1993.

[9] S. Gähler, Linear 2-normerte Raume, Math.Nachr, 28 (1965), 1-45. 
[10] K. Hensel, Theorie der Algebraischen Zahlen, Teubner, (Leipzig, 1908).

[11] S. Katok, Real and $p$-adic analysis course notes for math 497C Mass program, Fall 2000 Revised, The Pennsylvania state university, University Park, PA 16802, U.S.A. November 2001.

[12] N. Koblitz, $p$-adic Numbers, $p$-adic analysis, and zeta-Functions, 2nd ed. New York: Springer-Verlag, 1984.

[13] Ç.K. Koc, A Tutorial on $p$-adic arithmetic, Electrical\&Computer Engineering, Oregon State University Corvallis, Oregon 97331, Technical report, april 2002.

[14] M. Kurt, Introduction to $p$-adic numbers and their functions, 2nd ed. Cambridge, England: Cambridge University Press, 1981.

[15] Z. Lewandowska, Generalized 2-normed spaces, Stuspskie Prace MatematycznoFizyczne, 1 (2001), 33-40.

[16] Z. Lewandowska, Linear operators on generalized 2-normed spaces, Bull. Math. Soc. Sci. Math. Roumanie (N.S.), 42(90) (1999), no. 4, 353-368.

[17] Z. Lewandowska, On 2-normed sets, Glasnik Mat. Ser. III, 38(58) (2003), no. 1, 99-110.

[18] A.M. Robert, A course in p-adic analysis, Graduate texts in mathematics, vol. 198 (2000).

[19] V.S. Vladimirov, I.V. Volovich, E.I. Zelenov, p-adic analysis and mathematical physics, World scientific, Singapore, 1994.

[20] A. White, 2-Banach spaces, Math Nachr., 42 (1969), 43-60.

[21] S.S. William, Notes on p-adic numbers, Rice University, Houston, Texas. [arXiv:math/0502560v1[Math.CA] 26 Feb 2005.]

\author{
MeHMET AÇıKGöz \\ Department of Mathematics \\ Faculty of Science and Arts \\ University of GazianteP \\ 27310 GaZIANTEP \\ TURKEY \\ E-mail address: acikgoz@gantep.edu.tr
}

\section{Nurgül Aslan}

AdDress IS THE SAME FOR All aUthors

E-mail address: guzguzelim27@hotmail.com

\section{NuRTEN KÖŞKEROĞLU}

ADDRESS IS THE SAME FOR ALL AUTHORS

E-mail address: karsu_nurten@hotmail.com

\section{Serkan Araci}

AdDRESS IS THE SAME FOR ALL AUTHORS

E-mail address: mtsrkn@hotmail.com 